\title{
El espacio público en la ciudad: Una aproximación desde los precios hedónicos ${ }^{1}$
}

\author{
The public space in the city: \\ An approximation from the hedonic prices \\ O espaço público na cidade: \\ Uma abordagem de preços hedónicos
}

\author{
Alex Smith Araque Solano ${ }^{2}$ \\ Profesor Universidad Sergio Arboleda \\ alex.araque@correo.usa.edu.co \\ Julián Alberto Gutiérrez López ${ }^{3}$ \\ Grupo de Estudios y Análisis de la Defensoría del Espacio Público de Bogotá \\ jgutierrez@dadep.gov.co \\ Lina Fernanda Quenguan López ${ }^{4}$ \\ Grupo de Estudios y Análisis de la Defensoría del Espacio Público de Bogotá \\ lquenguan@dadep.gov.co
}

Recibido: 30/01/2017

Aprobado: 24/05/2017

\section{Resumen}

Esta es una investigación aplicada al sistema de parques de Bogotá, la cual tiene como objetivo proponer un modelo econométrico de determinación del valor del suelo del espacio público. Dada la complejidad para valorar el suelo público, la investigación se desarrolla a partir de combinar las determinantes de la teoría de la elección social por medio del teorema de la imposibilidad de Arrow y el modelo de precios (haciendo énfasis en la estructura urbana). Frente a lo anterior, la Defensoría del Espacio Público de Bogotá ha obtenido, como principal resultado, la evidencia de que existe una alta valoración social del espacio público, poniendo como reto la incorporación de análisis económicos más refinados que describan las diferentes valoraciones que hace la sociedad del espacio público. Así mismo, se encontró que la presencia de espacios públicos en el contexto urbano influye altamente en la determinación de los precios del suelo privado.

\footnotetext{
${ }^{1}$ Investigación realizada en el Grupo de Estudios y Análisis de la Defensoría del Espacio Público de Bogotá.

${ }^{2}$ Magíster en Economía.

${ }^{3}$ Economista.

${ }^{4}$ Especialista en mercado y política de suelo en América Latina.
} 
Palabras clave: espacio público; bienes públicos; valoración económica.

\begin{abstract}
This is a research applied to the Bogota parks system, which aims to propose an econometric model to determine the value of the public space soil. Given the complexity of valuing public land, the research is developed from combining the determinants of social choice theory through Arrow's impossibility theorem and the price model (by emphasizing the urban structure). In view of the above, the Ombudsman of Public Space of Bogotá has obtained, as a main result, evidence that there is a high social value of public space, putting as a challenge the incorporation of more refined economic analyzes that describe the different evaluations society makes of the public space. Likewise, it was found that the presence of public spaces in the urban context highly influences the determination of private land prices.
\end{abstract}

Keywords: public space; public goods; economic valuation

\title{
Resumo
}

Este documento é o resultado de uma pesquisa aplicada ao sistema dos parques na cidade de Bogotá, e tem como objetivo propor um modelo econométrico para determinar o valor do solo do espaço público. Dada a complexidade da valorização do solo público, a pesquisa foi desenvolvida combinando os determinantes da teoria da escolha social por meio do teorema da impossibilidade de Arrow e do modelo de preços (enfatizando a estrutura urbana). A Defensoria do Espaço Público, de Bogotá, conseguiu como resultado principal, a evidência de que existe um alto valor social do espaço público, exigindo a incorporação de análises econômicas mais refinadas para descrever os diferentes critérios de valor que a sociedade faz do espaço público. Verificou-se também que a presença de espaços públicos no contexto urbano, influenciou fortemente a determinação dos preços do solo privado.

Palavras-chave: espaço público; bens públicos; avaliação econômica.

\section{Introducción}

El espacio público, en sentido estricto, no tiene precio de mercado pero sí un costo de producción. Por su parte, los espacios privados internalizan en sus precios la presencia o cercanía de los espacios públicos. Las preferencias por este tipo de bienes posibilitan que el costo de producción del bien público no converja con la suma de las disponibilidades a pagar de los individuos localizados en su entorno inmediato. Establecer un precio de los bienes públicos requiere aclarar la relación de complementariedad de los bienes públicos y los bienes privados y lo pagado por la cercanía a un bien público local $(\mathrm{BPL})^{5}$.

En el estudio de las ciudades, urbanistas y economistas convergen en que lo público es un tema central. "El espacio público como tal es la ciudad, las ciudades son el ámbito

\footnotetext{
${ }^{5}$ Ahora bien, los recursos pagados por el bien público son apropiados por los dueños del suelo de forma que el constructor se queda con la ganancia normal.
} 
donde la población se reúne, se identifica allí se da forma a la expresión social" (Carrión, 2011 , p. 1). El espacio público funciona como un mecanismo indispensable en la estructuración de la ciudad y tiene como fin principal soportar el bienestar y la convivencia de las personas (Gehl, 2006); "los espacios como la plaza, la calle, el parque, los separadores, los andenes son elementos primordiales frente a las lógicas y dinámicas urbanas, para recuperar la ciudad como un conjunto" (Gamboa, 2003, p. 17).

Ahora bien, si el urbanismo es el vínculo de elementos, escuelas y normas que se aplican para que la cimentación y su asociación fomenten el desarrollo y acrecienten el bienestar individual, entonces viabiliza y garantiza la ciudad al construir y transformar espacio público permitiendo la continuidad, la conectividad y la articulación de todos los elementos que la componen (Cerdá, 1867).

En esta conceptualización, la ciudad se configura con el espacio público y este toma forma como función social en el territorio. Desde la economía espacial la ciudad es un agregado de bienes públicos que soporta la localización de la población y la realización de las diferentes actividades económicas (Fujita, Krugman \& Venables, 2000). El mercado no tiene un papel protagónico, la asignación de usos, por ejemplo, tiene como función el bienestar de la colectividad y este es distinto a la sumatoria de los intereses de productores individuales. La regla de preferencia social a partir de preferencias individuales sobre el conjunto social es lo que se conoce como la imposibilidad de Arrow, se requiere de los precios de Lindahl para corregir las propias distorsiones del mercado, en los cuales la cantidad pagada por cada individuo por el consumo de un bien específico sea igual al beneficio marginal que obtiene del mismo.

En el espacio público la corrección es a la inversa, no se incorporan las externalidades en los costos de producción, al contrario, los precios del espacio edificado son, por hipótesis, superiores a los precios de producción y los efectos externos de los bienes públicos son capitalizados por los propietarios del suelo y por los promotores inmobiliarios. De hecho, las preferencias de los individuos son la base de las decisiones de localización y se revelan con los precios de los espacios edificados en el entorno del espacio público, aquí el pago social es superior al costo de producción.

\section{Los bienes públicos y el mercado inmobiliario}

La inclusión de los bienes públicos en el análisis de utilidad de los individuos ha partido de sus características de no rivalidad y no exclusión, que los distinguen claramente de aquellos privados. Samuelson y Nordhaus ${ }^{6}$ sumaron a estas características la de indivisibilidad e incorporaron la producción en la función de utilidad de más de un individuo (Samuelson \& Nordhaus, 2010); mientras que Buchanan (1965) desarrolló la

\footnotetext{
6 En la práctica se da una gradación infinita o muy grande de las características de rivalidad y exclusión, de manera que muchos bienes comparten características públicas y privadas. Como lo son los servicios públicos domiciliarios o de servicios educativos y de salud que reciben entonces calificativos especiales como "meritorios" o "preferentes".
} 
teoría de los "bienes club", solo para un cierto conjunto de individuos ${ }^{7}$, de manera que limita su uso al pago de una membresía. Esta última característica define el carácter público del bien, donde su óptimo se configura a partir de la maximización de la utilidad del grupo de socios, la cual está dada en términos de la utilidad marginal de cada socio, y el costo deseable del bien será determinado por el acuerdo de consumo compartido.

Los bienes públicos enfrentan el problema de la revelación de las preferencias por parte de los consumidores; cualquiera sea la valoración que un agente haga de él no puede impedirse su consumo. Tiebout (1956) mostró que en una economía urbana la revelación de las preferencias se da en términos espaciales si se tienen en cuenta los gustos de los consumidores y las características locales de estos bienes. Esta noción implica que los BPL tienen un alcance limitado espacialmente el cual permite revelar las preferencias a partir de las decisiones de localización individual. En este modelo, si la oferta de bienes públicos se da a nivel central, los consumidores no tienen alternativas de elección en términos de localización al momento de nacer, los individuos a lo largo de su vida pueden adaptarse a las condiciones donde se nace o cambian de localización de acuerdo con su función de utilidad.

Las regiones compiten por captar población y, a su vez, por maximizar la función de utilidad de sus habitantes mediante la oferta de bienes públicos de forma que puede alcanzarse un equilibrio competitivo que cumple las condiciones de Pareto ${ }^{8}$. En estas condiciones la renta del suelo es indicador de las preferencias, por cuanto captura los incentivos para localizarse y la disponibilidad a pagar por bienes públicos en el sentido de Tiebout. De aquí la importancia del concepto de bien público local (BPL), el cual hace parte de la teoría del uso del suelo urbano que, a su vez, forma el núcleo de la economía urbana (Fujita, 1991).

En un modelo de ciudad basado en BPL, los beneficios del desarrollo urbano se expresan como incrementos en el precio del suelo con fuente en BPL, y en la generación de rendimientos crecientes por efecto de la aglomeración de actividades urbanas. Estos incrementos se estiman como rentas diferenciales a partir de un punto global de referencia (Fujita, 1991). El financiamiento de los BPL iguala la sumatoria de las rentas diferenciales con el costo de los BPL. De esta manera, con un tamaño óptimo de la población, las externalidades producidas por los BPL igualan las rentas diferenciales del suelo y los costos de producción de los $\mathrm{BPL}^{9}$. Si la población está por encima (debajo) del nivel óptimo, las rentas diferenciales serán mayores (menores) (Fujita \& Thisse, 2013). Lo anterior es conocido como el teorema George-Hotteling-Vickrey (teorema GHV) desarrollado hacia 1977 simultáneamente por Vickrey, Starret, Henderson y otros, con

\footnotetext{
7 Las áreas comunes de un conjunto cerrado, por ejemplo.

${ }^{8}$ El concepto de óptimo de Pareto hace alusión a una situación en la que no es posible beneficiar a una persona sin perjudicar a otra.

9 Se define "óptimo" como la correspondencia entre el nivel de servicios de los BPL y el tamaño de la población.
} 
antecedentes en los trabajos de Tiebout, Hotelling, Vickrey y George como lo menciona González (2006, p. 86).

Bajo esta noción, el mercado inmobiliario enfrenta tres tipos de precios en forma simultánea. El primero es el de la tierra rural, el cual es un dato de partida. El segundo es el precio de la tierra urbanizada que se encuentra determinado por los BPL necesarios en un modelo de costos de ciudad que puede estimarse a partir de su costo y cierta estructura de rentas diferenciales. $Y$ en el tercero, se tiene el precio final de subasta que incorpora las rentas diferenciales por externalidades de BPL y la renta en sentido estricto, determinada como renta de subasta, condicionada a su vez por la estructura de rendimientos crecientes y la demanda efectiva.

\subsection{Provisión eficiente de un bien público local}

Cada espacio edificado es producido con un componente de BPL, pero en toda situación urbana no necesariamente ocurre esto. En la producción y distribución de estos bienes no es posible lograr las condiciones de igualdad entre las tasas marginales de sustitución y las tasas marginales de transformación, debido a la característica de consumo conjunto y también a la presencia de rendimientos crecientes. Esto lleva a considerar la provisión de BPL por cuenta de monopolios públicos o privados, sin alcanzar la eficiencia vía precios o tarifas.

Un vector de BPL entra en la función de utilidad del hogar como uno de sus determinantes. Su efecto se expresa como externalidad, de manera que su financiación no entra directamente en el objetivo de maximización de la utilidad (beneficio). La financiación de los BPL debe hacerse como su consumo, es decir colectivamente, aunque en forma diferencial. Como ejemplo se propone el caso de una economía con dos agentes y dos bienes, uno de los cuales es un bien privado, xi, y el otro un bien público, gi. El ingreso del individuo se destina al consumo de los dos bienes, de forma que:

$w_{i}=x_{i}+g_{i}$

O:

$x_{i}=w_{i}-g_{i}$

Es decir, el gasto en bien público reduce el consumo del bien privado. La utilidad es creciente respecto del consumo de ambos bienes, la función se puede expresar como:

$u_{i}=\left(G, x_{i}\right)$

Si el bien público se oferta de forma discreta y su costo es "c", la tecnología se puede expresar por:

$G=\left\{\begin{array}{l}1 \text { Si } g_{1}+g_{2} \geq c \\ 0 \text { Si } g_{1}+g_{2}<c\end{array}\right\}$ 
Es eficiente producir el bien público, en el sentido de Pareto, si la suma de aportaciones supera el costo de producción. De esta manera:

$u_{1}=\left(1, w_{1}-g_{1}\right)>u_{1}=\left(0, w_{1}\right)$

$u_{2}=\left(1, w_{2}-g_{2}\right)>u_{2}=\left(0, w_{2}\right)$

Sea $r_{i}$ la cantidad mínima que está dispuesto a ofrecer el individuo "i" para la producción del bien público, o su precio de reserva. Por definición $r_{i}$ debe satisfacer la siguiente ecuación:

$u_{i}=\left(1, w_{i}-g_{i}\right)=u_{i}\left(0, w_{i}\right)$

Es decir, es una mejora, en el sentido de Pareto, suministrar un bien público discreto cuando la suma de las disposiciones a pagar es superior al costo de la provisión. En el caso en que el bien sea continuo la cantidad ofrecida viene dada por:

$G=f\left(g_{1}+g_{2}\right)$

La utilidad del individuo $i$ está dada por:

$U_{i}=f\left(\left(g_{1}+g_{2}\right), w_{i}-g_{i}\right)$

Al introducir la función de producción en la función de utilidad y obtener las condiciones de primer orden para la eficiencia, pueden hallarse maximizando la suma ponderada de las utilidades correspondientes a las variables $g_{1}$ y $g_{2}$, así:

$a_{1} \frac{\partial u_{1}\left(G, x_{1}\right)}{\partial G}+a_{2} \frac{\partial u_{1}\left(G, x_{1}\right)}{\partial G}=a_{1} \frac{\partial u_{1}\left(G, x_{1}\right)}{\partial x_{1}}$

$a_{1} \frac{\partial u_{1}\left(G, x_{1}\right)}{\partial G}+a_{2} \frac{\partial u_{1}\left(G, x_{1}\right)}{\partial G}=a_{2} \frac{\partial u_{2}\left(G, x_{2}\right)}{\partial x_{2}}$

Por tanto, $a_{1} \frac{\partial u_{1}\left(G, x_{1}\right)}{\partial x_{1}}=a_{2} \frac{\partial u_{2}\left(G, x_{2}\right)}{\partial x_{2}}$.

Si se dividen los primeros miembros de (10) por los segundos y se utiliza la igualdad se tiene que:

$\frac{\frac{\partial u_{1}\left(G, x_{1}\right)}{\partial G}}{\frac{\partial u_{1}\left(G, x_{1}\right)}{\partial x_{1}}}+\frac{\frac{\partial u_{2}\left(G, x_{2}\right)}{\partial G}}{\frac{\partial u_{2}\left(G, x_{2}\right)}{\partial x_{2}}}=1$

Es decir,

$R M S_{1}+R M S_{2}=1$ 
La condición de eficiencia en el caso de la provisión continua del bien público es que la suma de las disposiciones marginales a pagar sea igual al costo marginal de la provisión. En este caso el costo marginal es 1, ya que el bien público es simplemente la suma de las aportaciones. Como se aprecia en las últimas dos ecuaciones, un bien público se financia de acuerdo con las disposiciones marginales a pagar, pero en nuestras ciudades un individuo se localiza cerca de un bien público si paga el precio de mercado por esta localización. Es decir, el BPL cambia su condición para convertirse en un bien de mercado, bajo la connotación de BPL club.

De otra parte, este cambio en la condición del BPL crea desajustes del mercado, capitalizados por los propietarios del suelo en los precios de venta. Es decir, los precios del espacio público pagado son superiores a los precios de producción, en virtud de que la valoración social es superior a los BPL, es decir, se presenta un caso de la imposibilidad de Arrow, por lo tanto, las sumas de las disponibilidades marginales a pagar son superiores al costo de producción del BPL como lo indicaría la ecuación (4).

\subsection{Los precios del suelo y los bienes públicos como cargas urbanísticas}

Para describir de manera simple la relación entre los precios del suelo y los bienes públicos locales, considérese la existencia de un sector de la ciudad en el cual se tiene un lote originario de área bruta igual a X1 metros cuadrados y de precio total igual a P1. Si la ciudad señala que en la zona se deben dejar cesiones de espacio público iguales a la mitad del área, el predio, resultante (X2 - área neta urbanizable) vale el mismo monto $\mathrm{P} 1$; de forma que se duplica el precio por unidad de área $\left(\mathrm{m}^{2}\right)$. Si la ciudad exige equipamientos y en general bienes públicos locales, su costo de producción incrementará el precio del suelo en virtud de que se requiere producir suelo urbano. De manera explícita, la ecuación de precios y abstracción económica se puede presentar de forma menos compacta como:

$$
\begin{aligned}
& p a_{i j}(h)(1+r)+w l_{1}(h)+t(h) \rho(h)=p_{k} \mathrm{~h}=1 \ldots . \mathrm{K} \\
& \prod_{h=1}^{k} \rho_{k}=0
\end{aligned}
$$

Donde, $p$ corresponde al vector de precios, $a_{i j}$ los insumos, $r$ el beneficio del productor, $w$ el salario, $/$ el volumen de trabajo empleado, $t$ el área de suelo y $\rho$ la renta del suelo en los distintos suelos en los distintos suelos de productividad $h$.

La ecuación (14) indica que uno de los suelos no produce renta. Supóngase un momento en el cual un predio de área A de uso agrícola va a ser incorporado a la ciudad. Este tendrá un precio inicial derivado de las rentas agrícolas y de las presiones de urbanización en el margen urbano ${ }^{10}$, lo que exige que se introduzca en un proceso productivo para que

10 El propietario ya internalizó los costos de producción de suelo urbano y cobra el precio máximo para eliminar cualquier beneficio del constructor. 
actúe como un insumo urbano ${ }^{11}$. Es decir, las ecuaciones (13) y (14) tienen una modificación no insustancial que en la estructura urbana se nota con el incremento de la magnitud de las rentas urbanas:

$$
p a_{i 1}(h)(1+r)+w l_{1}(h)+t(h) \rho_{2}(h)=p_{k} \mathrm{~h}=1 \ldots . \mathrm{K}
$$

En esta aproximación todo suelo urbano es un suelo rural, al cual se le restan los costos de producción de la ecuación (15). En otras palabras, el suelo urbano entra como un costo adicional de producción en el primer término de la izquierda de la igualdad, pero se compensa con la reducción del valor de la renta del suelo. En tal virtud, al incorporar mayores dotaciones al suelo inicial, o bienes públicos locales, se tienen costos diversos. De esta forma, si:

$$
p a_{i 1}(1)(1+r)>p a_{i 1}(2)(1+r)>\cdots \cdot p a_{i 1}(h)(1+r)
$$

Entonces,

$$
p_{1}(1)>p_{1}(2) \ldots>p_{1}(h)(17
$$

Las rentas del suelo

$$
t(h) \rho(h)>0(18)
$$

Las rentas del suelo $t(h) \rho(h)$ se reducen por cuanto son función de las cargas urbanísticas. Esto significa que la incorporación de bienes públicos locales al suelo se entiende como costos de producción de suelo urbano de distintas calidades o condiciones y se restan de las rentas del suelo, en virtud de que el precio que paga el consumidor final no se modifica. Nótese que en el suelo $h$ de la ecuación (18), solo se desarrollarán las actividades $\varphi_{h}$ si el suelo tiene incorporados los costos que permiten desarrollar esta actividad de forma eficiente. Desde esta perspectiva, el costo de producción de los bienes públicos locales y, en consecuencia, su precio de producción iguala su coste, pero en el precio final se captura la renta del suelo como efecto externo.

\subsubsection{Imposibilidad de Arrow}

La teoría de la elección social se ocupa de la agregación de preferencias individuales, uno de sus principios básicos es la función de bienestar social, planteada por Bergson (1938) $W\left(u_{1}{ }^{\prime} \ldots, u_{n}\right)$, en la cual la función $W$ depende de todas las variables $u_{1}$ a $u_{n}$ que intervienen en el bienestar social y $u$ representa la función de utilidad de todos los individuos. Por su parte, $W$ y $u$ son adoptadas de tal forma que estas hermanen los valores éticos de la sociedad y, de esta manera, se encuentra que la finalidad de Bergson es poder plantear las funciones $W, u$ y sus respectivas restricciones con el fin de maximizar la función de utilidad $W$. El carácter ordinal de dicha función de bienestar

11 La administración de la ciudad puede exigir la construcción de bienes públicos locales en predios sin urbanizar como requisito para su introducción en el proceso inmobiliario. 
social planteó la necesidad de incluir un análisis cardinal mediante la introducción de índices que permitieran realizar comparaciones interpersonales.

Arrow (1951) hizo énfasis en la forma de construir "una ordenación social de las situaciones alternativas alcanzables, a partir de un conjunto dado de ordenaciones individuales de dichas situaciones sociales, estando el método de construcción acorde con los juicios de valor de la racionalidad y soberanía de los ciudadanos" (p. 63). Es decir, una regla de preferencia social, construida a partir de las preferencias individuales sobre el conjunto social, en la cual los agentes pueden manifestar sus preferencias sobre los diferentes estados de la sociedad y, con dicha regla, la preferencia social queda definida a partir de las preferencias individuales. Pero, a partir de su razonamiento, Arrow planteó que no existe un modelo de elección social con al menos tres agentes y tres alternativas y cuyas reglas de elección satisfagan las condiciones de dominio universal, condición fuerte de Pareto, independencia de las alternativas irrelevantes, no existencia de dictador y "representabilidad transitiva".

Sea una sociedad con $n$ individuos que definen sus preferencias sobre un conjunto de alternativas $X$. Suponiendo que existe una regla de elección social $C$ que verifique las condiciones expuestas por Rodríguez y Hernández (1996):

I. Racionalidad colectiva (U): la regla de elección social $f($.$) debe prescribir una$ ordenación $=f((>i) i \Omega I)$ sobre los estados sociales $X$ para toda colección de preferencias individuales $((>i) i \epsilon I) \epsilon I \Omega$.

II. Independencia de alternativas irrelevantes (I): sean $x$ e $y$ en $X$. La ordenación social de $x$ e y no varía con los cambios en la serie de ordenaciones hechas por los consumidores en otros estados sociales. Esto es, si $(>i) Y(>$ ' $i$ ) son series de ordenaciones de los consumidores que satisfacen $x>-i y$ $x>$ ' $i$ y para todo $i$, entonces la ordenación social de $x$ e y es la misma en las dos situaciones.

III. Principio de Pareto (P): sea $((>i) i \epsilon i) \epsilon \Omega$. Sí $x>i$ y para todo $i \in I$, entonces $x>y$ donde $>=f((>i) i \epsilon I)$. Si a de un individuo no se opone ninguna preferencia contraria de otro individuo, esa preferencia se preserva en la ordenación social.

IV. Condición de no - dictadura (O): sea $((>i) i \epsilon I) \epsilon \Omega$. Ningún individuo $j$ es un dictador, en el sentido de que $x>_{j} y$ implique $x>j$ e implique $x>y$, sin importar cuáles puedan ser las preferencias de los restantes individuos, donde también $>=f((>i) i \epsilon I)$. (p. 14).

En efecto, por la condición fuerte de "Pareto", hay coaliciones globalmente decisivas: al menos la totalidad de agentes lo será. Sea $S$ una coalición decisiva cuyo número de agentes sea mínimo; como $C$ no es dictatorial, $S$ debe tener al menos dos elementos. Elijamos un elemento $\{i\}$ en $S$. Notemos que $S-\{i\}$ sigue siendo no vacío. Consideremos tres alternativas (distintas dos a dos): $x, y, z$. Consideremos ahora un perfil $u$ que restringido al conjunto $\{x, y, z\}$ verifique que el agente $\{i\}$ cumple: $x P y, y P z$, todos los 
agentes de $S-\{i\}$ cumplen: $y P z, z P x$ y los agentes que no pertenecen a $S$ cumplen: $z P x, x P y$.

En la tabla 1 se presenta de forma esquemática.

Tabla 1. Coaliciones globalmente decisivas

\begin{tabular}{|c|c|c|}
\hline Sociedad & \multicolumn{2}{|c|}{} \\
\hline \multicolumn{2}{|c|}{$\boldsymbol{S}$} & \multicolumn{1}{|c|}{$\boldsymbol{s}$} \\
\hline$y-\{i\}$ & $\{i\}$ & $x P y$ \\
$z P z$ & $y P z$ & $x P y$ \\
\end{tabular}

Fuente: Tomado de Rodríguez y Hernández (1996).

Se deduce entonces que la sociedad tiene las siguientes preferencias:

1) $y \boldsymbol{P} Z$

Esto se deriva del hecho de que $S$ sea globalmente decisiva y todos sus individuos verifiquen que $y P z$.

2) $x \boldsymbol{R} y$

Esto se prueba por reducción al absurdo. Si la sociedad cumpliese que yPx y se aplica independencia de alternativas irrelevantes, se verificaría que $S-\{i\}$ es localmente decisiva para $y$ frente a $x$ (ya que verifican $x P y$ mientras que la sociedad cumple $y P x$ ). Teniendo en cuenta ahora el teorema anterior, $S-\{i\}$ sería globamente decisiva para $y$ frente a $x$. Lo cual entra en contradicción con el hecho de que $S$ es una coalición decisiva mínima.

3) $x \boldsymbol{P} z$.

Esto se deduce sin más que aplicar la propiedad transitiva a (1) y (2): como la sociedad cumple $x R y, y P z$ deducimos que $x P z$. Además, no puede ocurrir que la sociedad cumpla $z P x$, ya que si esto fuese cierto, junto con (2) $x R y$ se deduciría que $z P y$ (transitividad), lo que entraría en contradicción con (1) yPz.

De todo esto deducimos que $\{i\}$ es localmente decisiva para $x$ frente a $z$ (ya que para $\{i\}: x P y, y P z$ así que por la transitividad se cumple que $x P z$; por otro lado, para todos los agentes de $S-\{i\}$ y $S$ se tiene que: $z P x$; mientras la sociedad cumple: $x P z)$. Aplicando el teorema anterior sabemos que $\{i\}$ es globalmente decisiva para cualesquiera dos alternativas. Es decir que $\{i\}$ es un dictador y contradice las hipótesis de que $C$ era una regla no dictatorial (Rodríguez y Hernández, 1996).

\section{El modelo de precios y estructura urbana}


El modelo de determinación del precio del suelo del espacio público requiere encontrar los determinantes de los precios del espacio edificado más allá de su precio de producción, es decir, desagregar los efectos externos incorporados. La armonización de la ecuación (13) con la renta ofrecida indica que en cualquier suelo h se desarrolla la actividad económica que pueda ofrecer la mayor renta del suelo. La renta de equilibrio en cualquier punto de la ciudad es entonces expresada por:

$$
R^{*}(r) \equiv \max \left\{\max \Psi_{i}(r), 0\right\}
$$

Es decir, la envolvente de las máximas rentas ofrecidas en las distintas actividades urbanas. La renta del suelo es un residuo de la distribución del precio entre los agentes productivos, reclamado por los propietarios del suelo. "En un lugar de la ciudad se pueden ofrecer distintos espacios edificados con distintas rentas del suelo, en los terrenos de cruce de distintos gradientes de renta se puede desarrollar cualquier actividad. Si se desarrollan dos actividades vinculadas tecnológicamente estas ofrecerían la misma renta del suelo" (Fujita \& Thisse, 2013, p. 11). Por lo tanto, el aspecto que enmarca la localización de hogares y empresas es como lo indica la ecuación (19).

Siendo $\varphi_{1}(r$, ou $)$ la actividad que ofrece una mayor renta del suelo, esta se localizará en las proximidades del centro donde se formará una alta concentración de empleo. Por otro lado, en las localizaciones de $\varphi_{3}(\mathrm{r}$, ou $)$ habrá una menor concentración de empleo dada su lejanía al centro; "se supone que altos precios del suelo generados por las demandas del suelo exigen aprovechar al máximo el suelo de forma que la aglomeración de empleo desciende en el gradiente descrito por la envolvente diversas rentas diferenciales" (Alonso, 1964, p. 116), marcada por la ecuación (19). En este sentido, se necesita un modelo de precios que permita estimar el aporte de factores determinantes del precio del espacio construido como se señala en la siguiente ecuación.

$P E E^{i}=f(V E U, V E L, A P, \mu)$

Los precios del espacio edificado, $P E E^{i}$, es una función de factores de entorno urbano, VEU, de entorno local, VEL, y de atributos propios, AP, así como de eventos aleatorios, pero no estructurales $(\mu)$. En resumen, se estiman variables de estructura urbana, variables de estructura local, atributos propios y fenómenos particulares.

$$
V E U^{i}=f\left(D_{c}, D_{c t l d}, D_{\text {eq }} \text { metrop }, \text { Jerarquia }_{i}\right)
$$

Dentro de las variables de entorno urbano, la distancia al equipamiento metropolitano más cercano (Deqmetrop) se considera relevante, la distancia al centro (Dc) como explicativa de su localización dentro del gradiente urbano y la distancia a la centralidad más cercana $(D c t l d)$, permite capturar posibles distorsiones del gradiente de precios urbano por la existencia de múltiples centros (figura 1) y, finalmente, la jerarquía califica el tipo de centralidad a la cual pertenece la manzana. La teoría de los lugares centrales 
señala la importancia de un único centro, pero en las ciudades modernas este modelo es demasiado precario. De hecho, la expansión conlleva al incremento de los costos de desplazamiento y con ello las condiciones perfectas para la aparición de un nuevo lugar al cual acudir para la adquisición de los bienes necesarios. Esta situación plantea la jerarquización de cada centro, lo cual enmarcaría su funcionalidad económica y, a su vez, los precios del suelo indicarían la jerarquía de dicho centro, derivado de las mayores rentas ofrecidas, así como lo sería la concentración del empleo (Bourdeau-Lepage \& Hurriot, 2005).

Figura 1. Precios del suelo de una ciudad policéntrica

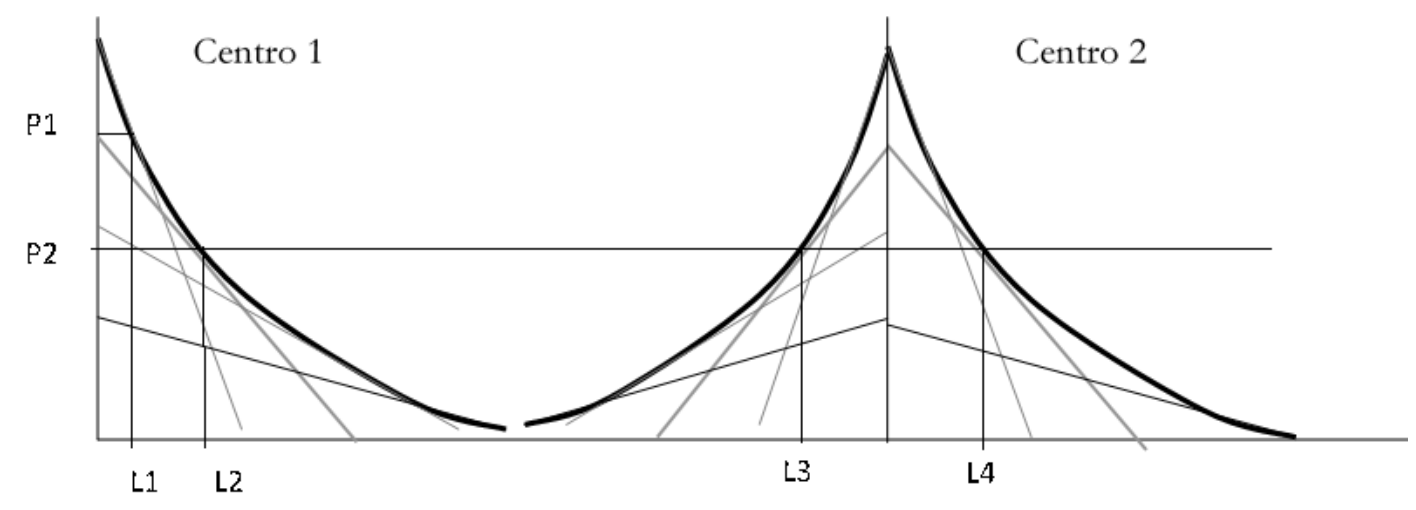

Fuente: Elaboración propia.

En este sentido puede ocurrir que el distrito central de negocios (DCN) se cualifique, lo que implica la expulsión de actividad de menor jerarquía hacia otros lugares urbanos donde su localización se adecua con la renta que pueden ofrecer por estar allí. Estos lugares aparecen en el escenario urbano en función de la distancia al DCN y la malla vial como determinante fundamental de la accesibilidad urbana y de allí de la localización empresarial (anexo 1. Centralidades de empleo de Bogotá); por ende, estos segundos centros tendrán una jerarquía menor. Por su parte, un segundo evento puede ocurrir cuando el DCN no ofrece las condiciones adecuadas para algunas actividades de gran jerarquía, lo que presiona la aparición de un nuevo centro que compite con el anterior por la supremacía urbana.

$$
V E L^{i}=f\left(A c c, D_{\text {equip }}, \operatorname{Den}_{m z}, D_{E E P}\right)
$$

Las variables de entorno local son la accesibilidad $(A c c)$, la distancia al equipamiento local $\left(D_{\text {equip }}\right)$, la densidad poblacional de la manzana $\left(D e n_{m z}\right)$, la distancia a la estructura ecológica principal $\left(D_{E E P}\right)$ y una variable dummy que califica el estado de la estructura ecológica principal en: bueno, regular y mal estado $\left(D_{E E P}\right)$.

$$
A P^{i}=f\left(\text { Estrato, Dotacionales, } \text { Uso }_{\text {prin }}, \text { Estado }_{E E P}\right)
$$


Las variables consideradas atributos propios son: el estrato, la distancia a los dotacionales cercanos, el uso principal de la manzana (Uso prin) y el estado de la estructura

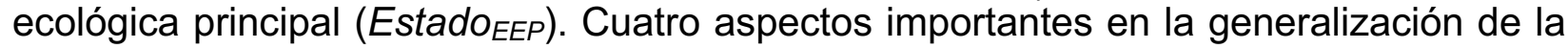
metodología para la cuantificación del valor del suelo público son: primero las distintas escalas de los espacios públicos, estas hacen difícil tener un efecto homogéneo sobre el precio del suelo. Segundo, la escasez de parques incrementaría el precio del suelo en el área anexa por la mayor valoración de los individuos; tercero, las distintas fracturas económicas del territorio que impiden hacer homogéneo el efecto en los precios del suelo debido a la ruptura de conectividad; y cuarto, la traslapación de las diferentes escalas de los parques por lo cual se debe considerar un mecanismo cuantificador. Para apreciar los efectos a escala se construyeron los polígonos de Thiessen ${ }^{12}$, como forma de establecer las áreas de influencia de cada parque en las escalas metropolitanas (figura 2), zonal (figura 3) y vecinal, local y parques menores (figura 4). En las siguientes imágenes se aprecian las distintas áreas de influencia:
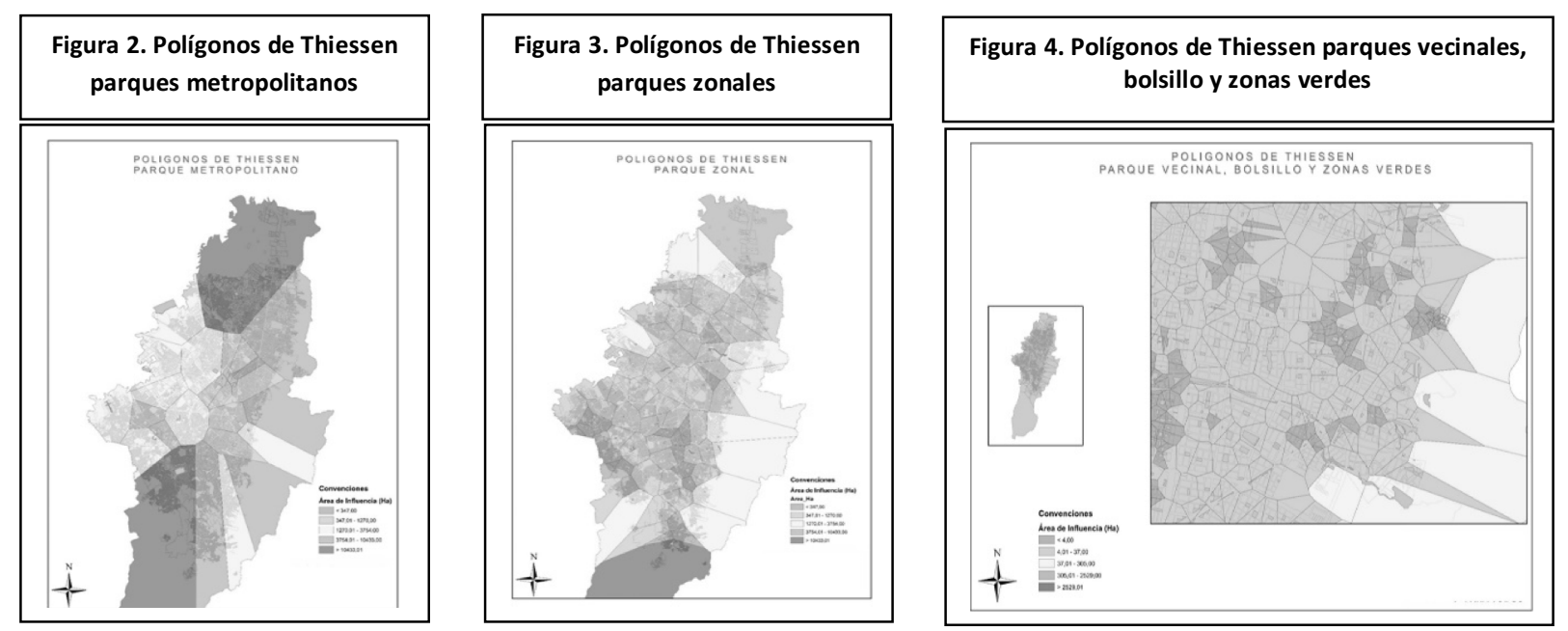

Fuente: Elaboración propia a partir de datos del Instituto Distrital de Recreación y Deporte y De la Defensoría del Espacio Público de Bogotá

Las distintas áreas sugieren este tipo de dificultades. En primer lugar, la escala metropolitana tiene un alcance más allá de su propia área de Thiessen. Por ejemplo, un parque metropolitano atrae población no solo de su entorno, sino también de toda la ciudad y de ciudades cercanas. El área de influencia de los parques locales cubre toda la ciudad de forma que el criterio de los 500 metros permite el traslape de las zonas de influencia de los anteriores y limita las distancias. Segundo, los polígonos no tienen en cuenta los accidentes geográficos y los urbanos. Un río, un cerro o una avenida limitan los efectos de los parques como ocurre en las dinámicas económicas con la localización

12 Para dos lecturas sobre el tema véanse:

http://datateca.unad.edu.co/contenidos/30172/MODULO\%20HIDROLOGIA/leccin_28_mtodo_de_poligon o_de_thiessen_y_de_isoyetas.html

http://support.esri.com/en/knowledgebase/GISDictionary/term/Thiessen\%20polygons 
del empleo, por ejemplo. Finalmente, otro problema es la escasez de parques, lo que hace aparecer un área extensa de afectación y, de esta manera, la accesibilidad al parque se diluye de forma que se sobreestima su influencia y el precio.

En este sentido, a pesar de que en los distintos modelos las escalas de los parques de gran alcance no salieron estadísticamente significativas, se tomó un conjunto de parques, del anexo 1, que permitiera aproximar un valor del suelo de este tipo de espacio público. Para hacerlo se toma como unidad de observación la unidad de planeamiento zonal (UPZ), en virtud de que su delimitación contempla los límites físicos y económicos en áreas más o menos homogéneas del territorio. Igualmente se tiene en cuenta el número de parques vecinales por UPZ y la frecuencia junto con el área agregada de los parques. Si este número de parques es igual a 1 y el área es menor a la media, se considera que la UPZ tiene escasez de parques de esta categoría.

Por lo tanto, en razón de la realización de un ejercicio econométrico con ofertas de vivienda en las revistas La Guía y Metro Cuadrado, se debe asumir un modelo de localización urbana de los hogares. De esta manera, si el consumidor se encuentra a una distancia, $r$, del distrito central de negocios, o centro de la ciudad, su ecuación presupuestaria está definida por:

$$
Y=z+s R(r)+T(r)(24)
$$

Donde $z$ es un bien compuesto de todos los bienes consumidos excepto suelo; $s$ es el tamaño del lote; $R(r)$ la renta del suelo pagada en su lugar de vivienda y $T(r)$ el costo de transporte incurrido para desplazarse de su lugar de residencia a su trabajo o viceversa. El problema de un consumidor tipo se establece en términos de su localización y de su ingreso y es:

$$
\operatorname{Max} U(z, s)
$$

Sujeta a $Y=z+s R(r)+T(r)$

Ó $Y-T(r)=z+s R(r)$

La renta ofrecida por el consumidor de espacio residencial será función de su nivel máximo de utilidad y la distancia al centro. El consumidor debe intercambiar consumo del bien compuesto por área de terreno o viceversa. Maximiza su utilidad cuando la recta de presupuesto iguale la tasa marginal de sustitución de los dos bienes o, lo que es lo mismo, la renta ofrecida y, por supuesto, sean tangentes a la curva de indiferencia. Si se usa el teorema de la envolvente se puede establecer, sin demasiadas complicaciones matemáticas, que:

$$
\frac{\partial \Psi(r, u)}{\partial r}=-\frac{T^{\prime}(r)}{S(r, u)}<0
$$




$$
\frac{\partial \Psi(r, u)}{\partial u}=-\frac{1}{S(r, u)} \frac{\partial Z(s, u)}{\partial u}<0
$$

Es decir, la renta ofrecida es continuamente decreciente en la distancia, $r$, y en la utilidad, $u$, hasta que se hace cero. De forma adicional, el tamaño del lote es continuamente creciente en $r$ y $u$, es decir, mayor distancia al centro implica un lote mayor, y mayor utilidad mayor área de lote.

\section{Modelo de precios de suelo de Bogotá}

El modelo inicial fue ajustado para incluir las interacciones de los distintos elementos del espacio público y de las dotaciones urbanas consideradas al momento de adquirir espacio edificado. En la compra no solo se incluye cercanía de parques, sino también cercanía a colegios, jardines infantiles, unidades de atención médica, centros de atención inmediata en seguridad, centros comerciales, entre otros ${ }^{13}$.

La ecuación estimada, en logaritmos, toma la distancia a la centralidad más cercana, I_dist_m_cc,la distancia al tradicional distrito central de negocios en la Jiménez con calle 13, I_dist_13, la distancia a la calle 72 como un nuevo distrito central de negocios, I_dist_72, la densidad de población, la densidad de empleo en el año 2005 como proxys de la densidad de población y empleo actual, I_dens_pob y l_dens_emp respectivamente, una combinación lineal de la población por edad, una combinación lineal de la educación de la población I_ind_educa, la distancia a los centros comerciales, I_dist_centrocomercial, una combinación lineal de la distancia a los equipamientos, I_f1, una combinación lineal de la distancia a los paraderos de bus, I_a1, y finalmente las interacciones de los bienes públicos básicos, I_int_bpl_basic.

Los resultados, donde todas las variables son estadísticamente significativas a 95\% de confianza se presentan en la tabla 2.

En la tabla se aprecia que a medida en que se aleja de la centralidad más cercana, "I_dist_m_cc", el precio se reduce muy poco. Es decir, al aumentar la distancia de 100 a 200 metros, $100 \%$, el precio del suelo se reduce en $1,4 \%$. Esto significa muy poca discriminación de precios de la centralidad. Una situación distinta ocurre con la calle 72, donde se aprecia la jerarquía del lugar en su coeficiente. Al incrementar la distancia cae rápidamente el precio del suelo. El signo positivo del antiguo distrito central de negocios sugiere la existencia de otros centros de superior jerarquía, que distorsionan el gradiente normal.

Las densidades de población y de empleo, "I_dens_emp" y "I_ind_pob" respectivamente, guardan consistencia con la evidencia empírica de la ciudad. Las zonas del margen urbano, donde se tienen las mayores densidades de población, tienen menores precios del suelo. Las zonas de empleo tienen mayor valor del suelo y su saturación indica mayor

13 Las variables de los modelos estimados se presentan en el anexo 2. 
jerarquía y, con ella, mayor precio del suelo. El indicador de población, "I_ind_pob", combinación lineal de las edades por grupos quinquenales, señala qué lugares de la ciudad donde solo se localiza población adulta tienen mayores precios. El ingreso del hogar, medido por la combinación lineal del nivel de educación "I_ind_educa", se asocia con un precio superior, a mayor educación mayor ingreso y, por lo tanto, mayor posibilidad de pago de una renta del suelo superior.

Tabla 2. Modelo de precios de suelo de Bogotá

\begin{tabular}{|c|c|c|c|c|}
\hline $\mathrm{N}^{\circ}$ de observaciones & $\mathrm{F}(11,24092)$ & Prob $>\mathrm{F}$ & R-squared & RMSE \\
\hline 24104 & 2443,7 & 0,000 & 0,653 & 0,383 \\
\hline Variables & \multicolumn{2}{|c|}{ Coeficientes } & \multicolumn{2}{|c|}{ Errores estadísticos } \\
\hline I_dist_m_cc ${ }^{* * *}$ & \multicolumn{2}{|c|}{$-0,01411$} & \multicolumn{2}{|l|}{0,00200} \\
\hline \multicolumn{2}{|l|}{ I_dist_13 ${ }^{* * *}$} & 0,27240 & \multicolumn{2}{|l|}{0,00623} \\
\hline I_dist_72 *** & \multicolumn{2}{|c|}{$-0,36551$} & \multicolumn{2}{|l|}{0,00848} \\
\hline I_dens_pob *** & \multicolumn{2}{|c|}{$-0,19719$} & \multicolumn{2}{|l|}{0,00754} \\
\hline I_dens_emp *** & \multicolumn{2}{|c|}{0,03542} & \multicolumn{2}{|l|}{0,00275} \\
\hline I_ind_pob *** & \multicolumn{2}{|c|}{$-0,36464$} & \multicolumn{2}{|l|}{0,03854} \\
\hline I_ind_educa *** & \multicolumn{2}{|c|}{0,53239} & \multicolumn{2}{|l|}{0,03680} \\
\hline I_dist_centrocomercial ${ }^{* * *}$ & \multicolumn{2}{|c|}{$-0,15067$} & \multicolumn{2}{|l|}{0,00503} \\
\hline I_f1 ${ }^{* * *}$ & \multicolumn{2}{|c|}{$-0,12623$} & \multicolumn{2}{|l|}{0,01019} \\
\hline I_a1 ${ }^{* * *}$ & \multicolumn{2}{|c|}{$-0,03831$} & \multicolumn{2}{|l|}{0,01110} \\
\hline \multicolumn{2}{|l|}{ I_int_bpl_basic ${ }^{* * *}$} & $-0,00811$ & \multicolumn{2}{|l|}{0,00071} \\
\hline cons $^{* * *}$ & \multicolumn{2}{|c|}{18,02714} & \multicolumn{2}{|l|}{0,11398} \\
\hline
\end{tabular}

Fuente: Elaboración propia.

El centro comercial tiene un efecto positivo en los precios de los suelos en el entorno del centro comercial. Al alejarse de este entorno el precio desciende. Una situación similar ocurre con los equipamientos "I_f1" y los accesos a los paraderos del sistema de transporte, I_a1. Las interacciones de bienes públicos básicos, "I_int_bpl_basic", sugieren que al incrementar la distancia a estos bienes el precio del suelo cae. Si la distancia pasa de una manzana a la siguiente, se supone un incremento del $100 \%$ y el precio cae en $0,0081116 \%$. Si en una zona con estos bienes el precio es de $\$ 1.000 .000$, pasar de una manzana a otra más lejana, el precio desciende a \$991.954.

\subsection{Aplicación de la metodología}

El Parque de la 93, ubicado entre las calles 93a y 93b y la carrera 11, está localizado en uno de los entornos urbanos de mayor valor económico de Bogotá (figura 5). En 1995, mediante la iniciativa privada y un gran porcentaje de recursos públicos ${ }^{14}$ y algunos privados, se renovó el parque a un costo de 1.516 millones de pesos de 2015. Su selección obedeció a varias razones. En primer lugar, es un ícono en la ciudad de alta

14 http://www.eltiempo.com/archivo/documento/MAM-280992 
jerarquía. En segundo lugar, se encuentra encerrado por vías que implican el rompimiento de la continuidad del gradiente de precios y, por supuesto, el mismo gradiente derivado del efecto del parque sobre los precios. En tercer lugar, si bien es un parque con las condiciones señaladas, es de carácter vecinal.

\section{Figura 5. Valoración Parque de la 93}

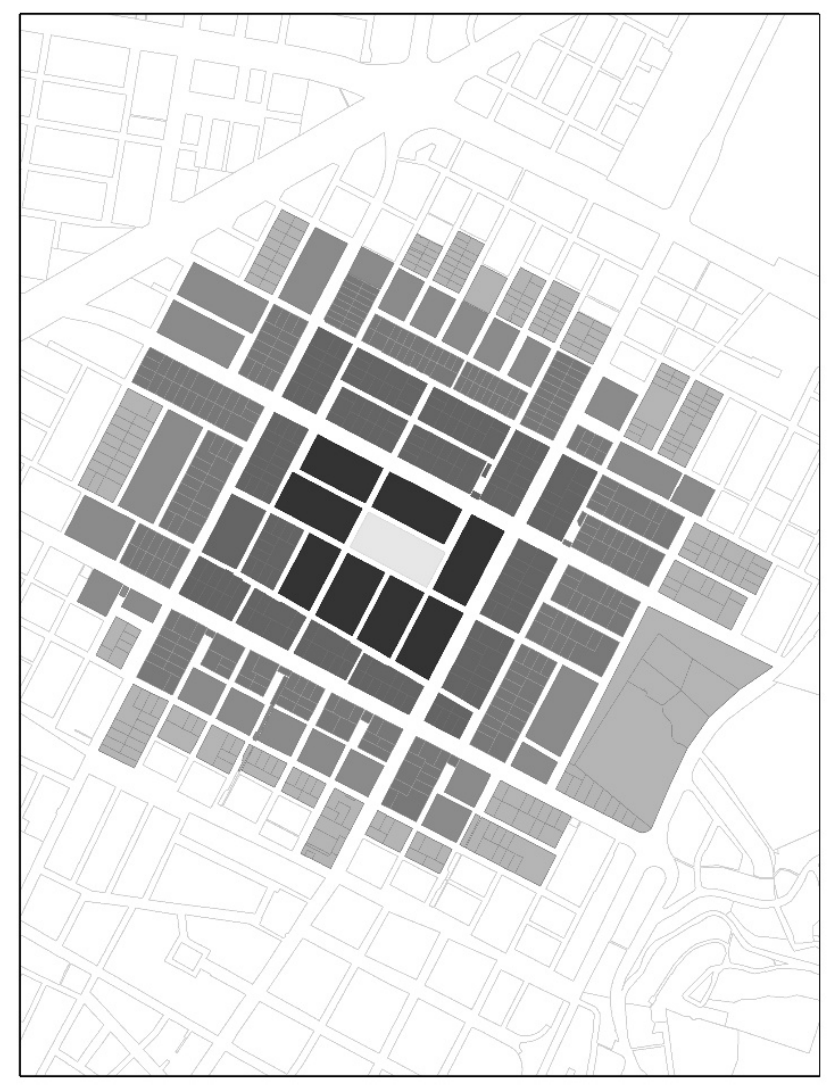

Fuente: Elaboración propia con información de la Unidad Administrativa Especial de Catastro Distrital.

De acuerdo con la metodología, los propietarios pagaron en millones de pesos, por su localización, en la primera línea de manzanas $\$ 7.686$, $\$ 14.473$ en la segunda, $\$ 15.837$ en la tercera, $\$ 11.955$ en la cuarta y $\$ 16.102$ en la quinta. Al sumar estos valores, el parque tendría un precio de $\$ 66.055$ millones para un área de 13.649 metros cuadrados, lo que se traduce en un precio por metro cuadrado de $\$ 4.605 .099$. El parque tiene un avalúo catastral de $\$ 15.481$ millones, y los costos de las construcciones alcanzan los $\$ 3.200$ millones. En este sentido, el metro cuadrado de suelo tiene un avalúo de $\$ 899.773$. Los predios privados de la zona tienen un avalúo medio de $\$ 3.910 .000$ el metro cuadrado. Es decir, la razón estimada del precio del metro cuadrado de parque a suelo privado pasa de 0,23 a 1,18 veces. 
Lo que significa que la razón precio de los dos tipos de suelo es de 0,23 por cuanto el suelo privado es más costoso. Con la metodología propuesta, la razón sube de forma representativa y alcanza el valor de 1,18 veces. El suelo de espacio público es $18 \%$ más costoso. Desde las dos perspectivas de este documento no puede el suelo del espacio público tener un valor menor que el privado. De hecho, si en una zona de la ciudad de parques inexistentes se demolieran edificaciones para dejar un parque de una manzana, el metro cuadrado tomaría el valor del costo del suelo del uso anterior.

En conclusión, si la UPZ tiene escasez de parques el precio del metro cuadrado será, al menos, del nivel de la razón encontrada en el ejercicio de aplicación en el Parque de la 93, superior en $18 \%$ al precio homogéneo del metro cuadrado de la zona anexa al parque. Si la zona tiene excesos de parque, el precio iguala a los precios homogéneos del área anexa al parque. En cualquier caso, el límite inferior del precio por metro cuadrado de parque iguala al precio homogéneo de los predios contiguos al parque. De hecho, se realizó un ejercicio en varias zonas de la ciudad y los precios de los predios de los lotes contiguos son muy homogéneos. En virtud de la heterogeneidad de algunos precios, se propone tomar para el precio el promedio ponderado de precios de cada manzana por cada uso para toda la ciudad.

\section{Conclusiones}

La estimación del precio del suelo de espacio público en las ciudades exige ir más allá de las técnicas avaluatorias e incorporar el análisis económico, de forma que se describan las distintas valoraciones que hace la sociedad de las variables urbanas y, por supuesto, del mismo espacio público. En primer lugar, no hay ciudades sin los espacios públicos, lo privado no hace ciudad de forma que la propiedad privada solo toma valor con lo público. En segundo lugar, se debe incorporar en la estimación la heterogeneidad urbana, accesibilidad, estructura urbana etc., para filtrar el valor neto del espacio público, como un agregado de las interacciones de factores que determinan la localización. Los individuos eligen una canasta de bienes públicos, no solo zonas verdes.

En nuestra opinión, es un error asignar un precio de lo público inferior al precio del suelo privado. No tiene justificación teórica o empírica. Se podría encontrar el origen de esta falla en las dificultades que encuentran los avaluadores al valorar bienes que no tienen mercado y solo costos de producción que pudieron ser históricos. De nuevo, señalar que el suelo de espacios públicos tiene un valor inferior al suelo homogéneo privado implica desconocer la propia estructuración de las ciudades.

Se esperaba una mayor suma por la valoración social del espacio público a la suma de las valoraciones individuales, sobre la base del teorema de la imposibilidad de Arrow, como en efecto ocurrió. Al respecto debe señalarse que, en estadios de desarrollo de terrenos iniciales, se conserva la imposibilidad frente a la valoración del área bruta y el área útil; la primera es inferior a la última y se podría señalar que contablemente este valor del suelo es inferior al suelo útil. De nuevo, el asunto no es así por cuanto los individuos valoran los bienes públicos y pagan una suma superior a su costo de 
producción. Si, como en el caso del Parque de la 93, su construcción y revaloración se dio cuando ya había una zona urbana consolidada, las inversiones realizadas vieron multiplicar el precio de las zonas aledañas de manera significativa. De nuevo, la valoración social es superior a la individual que planteó apenas inversiones pequeñas frente a su valor estimado.

Se requiere mejorar la incorporación de los efectos y traslapes de las distintas escalas de los parques para tener mayor claridad sobre el aporte de estos al precio. Es un ejercicio difícil en virtud de su localización, la cual está asociada a áreas de dotaciones urbanas de gran alcance de manera que requieren de accesibilidad y esto rompe la continuidad de valor para dejarlos como islas. Tal es el caso del Parque Simón Bolívar o el de Los Novios, por ejemplo.

\section{Referencias}

Alonso, W. (1964). Location and land use: Toward a general theory of land rent. Cambridge, US: Harvard University Press.

Arrow, K. J. (1951). Elección social y valores individuales. Madrid, España: Planeta-De Agostini.

Bergson, A. (1938). A reformulation of certain aspects of welfare economics. The Quarterly Journal of Economics, 52(2), 310-334.

Bourdeau-Lepage, L. \& Huriot, J. M. (2005). On poles and centers: Cities in the French style. Urban Public Economics Review, (3), 13-36.

Buchanan, J. M. (1965). An economic theory of clubs. Economica, 32(125), 1-14.

Carrión, F. (2011). Arq. La ciudad es el espacio público. Recuperado de https://works.bepress.com/fernando carrion/462/

Cerdá, I. (1867). Teoría general de la urbanización, y aplicación de sus principios y doctrinas a la reforma y ensanche de Barcelona (Vol. 1). Madrid, España: Editorial Imprenta Española.

Fujita, M. (1991). Urban economic theory. Cambridge, UK: Cambridge Books.

Fujita, M. y Thisse, J. (2013). Economía de aglomeración ciudades, Industriales Localización y globalización. Cambridge, Reino Unido: Cambridge University Press.

Fujita, M., Krugman, P. R. y Venables, A. J. (2000). Economía espacial: las ciudades, las regiones y el comercio internacional. Cambridge, Reino Unido: Ariel.

Gamboa, P. (2003). El sentido urbano del espacio público. Bitácora Urbano Territorial, 1(7), 13-18. 
Gehl, J. (2006). La humanización del espacio urbano: la vida social entre los edificios (vol. 9). Barcelona, España: Reverté.

González, J. (2006). La dimensión de lo razonable en la micro de William Vickrey (19141996). En Ética, economía y políticas sociales (pp. 57-102). Medellín, Colombia: Corporación Región.

Rodríguez, M. y Hernández, E. (1996). Elección social: teorema Arrow. Documento de trabajo. Universidad de Oviedo, Facultad de Ciencias Económicas y Empresariales. Recuperado de https://econo.uniovi.es/c/document_library/get_file?uuid=837d985f85d5-4a40-8470-8b731868d292\&groupld=746637

Samuelson, P. A. y Nordhaus, W. D. (2010). Macroeconomía con aplicaciones a Latinoamérica. Ciudad de México, México: McGraw-Hill.

Tiebout, C. M. (1956). Una teoría pura de los gastos locales. Diario de la economía política, 64(5), 416-424. 


\section{Anexo 1. Centralidades de empleo de Bogotá}

CENTRALIDADES

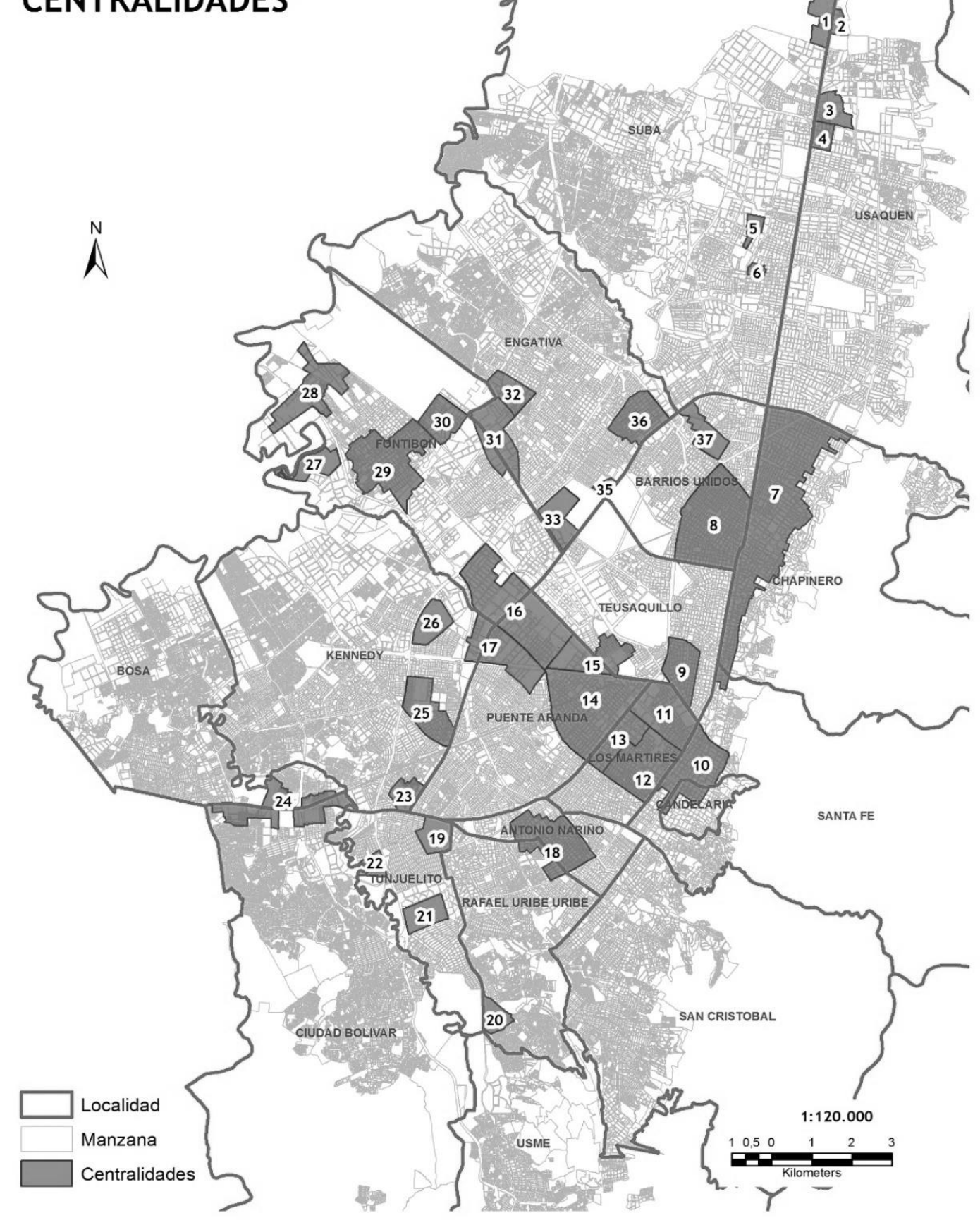

Fuente: Elaboración propia. 


\section{Anexo 2. Variables de los modelos estimados}

El nemónico de las variables utilizadas en el modelo son: dist_sitp, Distancia al paradero SITP, dist_parad, Distancia al paradero transporte, aval2009, Avalúo 2009, tot_est_mz, Total establecimientos por manzana en el año 2005, tot_emp_mz, Total empleo en la Manzana 2005, población, Población en la MZ 2005, dens_pob, Densidad de población, ind_pob, Índice de población, estrato_06, Estrato, ind_educa, Índice de educación de la población de la MZ en 2005, dens_emp, Densidad de empleo, dist_m_cc, Distancia al Centro empleo cercano, virzhge_2014, Valor de referencia 2014, virzhge__2013, Valor de referencia 2013, dist_parqu, Distancia al parque más cercano, dist_vias, Distancia a las vías, tip_via, Tipo de vía, dist_abas, Distancia al equipamiento más cercano de abastecimiento, esc_abas, Escala del equipamiento de abastecimiento, dist_equip_bs, Distancia al equipamiento más cercano de bienestar social, esc_eqbs_s, Escala del equipamiento de seguridad social, dist_eq_culto, Distancia al equipamiento más cercano de culto, esc_eq_cults, Escala del equipamiento de culto, dist_eq_cult, Distancia al equipamiento más cercano de cultura, dist_eq_educ, Distancia al equiámiento más cercano de educación, esc_eq_educs, Escala del equipamiento de educación, dist_rec_fer, Distancia al equipamiento más cercano de recintos feriales, esc_rec_fers, Escala del equipamiento de recintos feriales, dist_eq_rec_dep, Distancia al equipamiento más cercano de deportes, esc_eq_rec_deps, Escala del equipamiento de recintos deportivos, dist_eq_salud, Distancia al equipamiento más cercano de salud, esc_eq_saluds, Escala del equipamiento de salud, dist_eq_seg, Distancia al equipamiento más cercano de seguridad, esc_eq_segs, Escala del equipamiento de seguridad, dis_est_tm, Distancia a la estación de Transmilenio, dist_parques_ t, Distancia a los parques totales, dist_parque_b o, Distancia al parque de bolsillo, dist_esc_depo o, Distancia al escenario deportivo, dist_parque_v I, Distancia al parque vecinal, dist_parque_m o, Distancia al parque metropolitano, dist_parque_z I, Distancia al parque zonal, f1, Combinación lineal de equipamientos, dist_zonaverde, Distancia a la zona verde, dist_zonaverd I, Distancia a la zona verde comunal, dist_plaza, Distancia a la plaza, dist_plazoleta, Distancia a la Plazoleta, dist_cesionaa, Distancia a la cesión tipo A, d_500_dist_zo e, Dicotómica de existencia de zona verde en área de $500 \mathrm{~m}$, d_500_dist_zo I, Dicotómica de existencia de zona verde comunal en área de $500 \mathrm{~m}$, d_500_dist_p za Dicotómica de existencia de plaza en área de 500 m, d_500_dist_p ta, Dicotómica de existencia de plazoleta en área de $500 \mathrm{~m}$, dist72, Distancia a la calle 72 con carrera $7^{\mathrm{a}}$, dist13, Distancia a la calle 13 con carrera $7^{\mathrm{a}}$. 